\title{
Status, Distribution, and Diversity of Birds in Mining Environment of Kachchh, Gujarat
}

\author{
Nikunj B. Gajera, Arun Kumar Roy Mahato, and V. Vijay Kumar \\ Gujarat Institute of Desert Ecology, Mundra Road, P.O. Box 83, Bhuj, Kachch,, Gujarat 370001, India \\ Correspondence should be addressed to Nikunj B. Gajera; gajeranikunj@gmail.com
}

Received 4 December 2012; Revised 3 March 2013; Accepted 8 March 2013

Academic Editor: Rafael Riosmena-Rodríguez

Copyright (c) 2013 Nikunj B. Gajera et al. This is an open access article distributed under the Creative Commons Attribution License, which permits unrestricted use, distribution, and reproduction in any medium, provided the original work is properly cited.

\begin{abstract}
Opencast mining is one of the major reasons for the destruction of natural habitats for many wildlife including birds. The Kachchh region belongs to the arid part of India and is one of the rich areas of mineral resources in the country. In the recent time and after the 2001 earthquake, mining and other developmental activities are increased, and as a result, the natural habitats of birds are disturbed and fragmented. So, this study was conducted to assess the impact of mining and associated activities on the diversity and distribution of birds. Birds were studied by surveying 180 transects along 9 zones around three selected major mines, and each zone is made in every $2 \mathrm{~km}$ radius from the mine. Based on the record, it was found that the density and diversity of birds are highest in zone 5 and lowest in zone 1 and zone 2, respectively. The result indicates that the diversity and abundance of birds were less in zones which are located close to the mines in comparison to the zones far from the mines. In conclusion, mining and its associated activities have some impacts on the diversity and distribution of birds in Kachchh region in India.
\end{abstract}

\section{Introduction}

Mining and its related activities are one of the major causes for the destruction of natural habitats for wildlife. As these activities increased in context of rapid use of natural resources to meet the demand of the market and the development of region and country, the pressure of threat to the wildlife is increasing day by day. In addition to mining, large parts of forests and wildlife habitats are being cleared for agriculture, industry, roads and railways network, and human habitation leading to degradation, fragmentation, and loss of habitat contributing to the overall loss of biological diversity. The loss of habitat and biological diversity by means of mining and its associated activities creates imbalance in ecological equilibrium.

Kachchh is one of the rich avifaunal diversity areas of India and also falls under one of the migratory route of the avifauna of this country. The list of birds of Kachchh district was made by some workers from time to time; notably among them were Lester [1], Ali [2], Ripley [3], Himmatsinhii [4], and Maharao [5]. The Kachchh district supports around 303 species of birds as recorded by Tiwari [6] and 370 bird species as reported by Sen [7] with some species including raptors, waterfowl, waders, and larks which are commonly found in this district. Similar to that, Kachchh is also a rich area of minerals including limestone, bentonite, and lignite. After the 2001 earthquake in Kachchh, many steps like industrialization and mining activities were increased for the development of this region. The consequence of the above activities results in the loss and fragmentation of habitat for wildlife. The major impacts on avifaunal diversity by means of the above development in postearthquake period are not being studied and assessed. In addition to that, so far no studies were made on the status, distribution, and diversity of birds in relation to the impact of mining and other developmental activities.

The bird species are widely distributed among various habitats in western Kachchh region. Various factors associated with different habitat types had a distinct impact on bird species. Likewise, various land use activities especially opencast mining that is being done at a mass scale in the region also affect the bird populations considerably, so we tested the following research hypotheses. 
Ho: There is an impact of mining on the distribution and diversity of bird species.

\section{Materials and Methods}

2.1. Study Area. The study was conducted in three major mining areas, namely, Pandhro, Mata-nu-madh, and Jadva. The mining activities are carried out for extraction of minerals including limestone, bentonite, and lignite. All the three mines are opencast mines results into that major loss of habitats by extraction of minerals and dumping of waste materials in open ground. These mines are situated in the westernmost part of Kachchh district, Gujarat, and are very close to the Narayan Sarovar Wildlife Sanctuary, which is the only habitat in Kachchh for Indian gazelle (Gazella benetti) and some other wildlife. This area falls in the arid zone of the country and is characterized by presence of an admixture of distinguishable habitats such as Acacia forest (AF), Euphorbia salvadora forest (ES), mixed thorn forest (MTF), Prosopis forest (PF), dense grassland (DG), and sparse grassland (SG). Some seasonal and perennial wetlands and agricultural fields were also interspersed in and around the mining areas. The habitats found in the study area were broadly divided into seven habitat types which are described below.

Acacia Forest (AF). In this habitat, Acacia senegal and Acacia nilotica were dominant species with negligible distribution of P. juliflora.

Mixed Thorn Forest. This habitat is a complex distribution of species like Acacia nilotica, A. senegal, Euphorbia caducifolia, Grewia spp. Commiphora wightii, and Salvadora spp. with some individuals of $P$. juliflora.

Euphorbia salvadora Forest. This habitat, dominated by Euphorbia caducifolia, Salvadora persica, and Salvadora oleoides.

Prosopis Forest. This habitat dominated by the P. Juliflora (an Invasive alien species) with few other species like Grewia tenax, G. villosa, and Capparis deciduas which were also found.

Grassland Forest. Some areas were flat to gentle undulate terrain covered by annual or perennial grasses, and absence of shrub or tree species which were categorized under grassland. Grassland has a main component of the member of Gramineae family. Cymbopogon spp. and Dichanthium spp. are the major grass species.

Agriculture Field. This habitat included agriculture field (fallow or current agriculture) in which annually single cropping system was existed. The agricultural fields were also invaded by $P$. juliflora, Capparis deciduas, or Salvadora spp.

Wetland. Wetland habitat included man-made and natural water bodies with the presence of water in the whole year or some parts of the year. Most of the wetlands of the study area were man-made due to low precipitation rate.
2.2. Sampling Method. Prior to field surveys, the study area was stratified into various distinguishable habitats such as Acacia forest (AF), Euphorbia salvadora forest (ES), mixed thorn forest (MTF), Prosopis forest (PF), dense grassland (DG), and sparse grassland (SG). The above habitats were evenly distributed in the whole study area. After stratification, the entire area was divided into $5 \times 5 \mathrm{~km}$ grids using survey of India's georeferenced coordinate system. Those grid cells were further subdivided into $1 \times 1 \mathrm{~km}$ smaller grids, and a total of 180 transects were laid down randomly within the grids (Figure 1). The length of each transect was $1.1 \mathrm{~km}$ and was laid down randomly to cover each kind of habitats. To understand the impact of mining on birds, the diversity and distribution pattern of bird was recorded by dividing the area into nine circular zones (zone 1 to zone 9) with an interval of $2 \mathrm{~km}$ from the centre of mine (Figure 1). Care was taken to have adequate sampling in each of the topographical features across lateral and vertical gradients like altitudinal range and terrain, and spatially within each habitat type.

Bird survey was done using direct count methods which include (1) point count method and (2) area search method. In point count method [8], birds were recorded in four circular plots with $25 \mathrm{~m}$ radius in every $300 \mathrm{~m}$ distance along transects. In area search method [9], survey was made in $3 \mathrm{~m}$ wide belt along transect in between the circular plot. The time taken for survey varied depending on the terrain and topography. Surveys were conducted in the morning hours (6.30 a.m. to $9.30 \mathrm{a} . \mathrm{m}$.) and evening hours (3.30 p.m. to 6.30 p.m.) by a single observer. Samplings were made in seasonal basis for the period of three years (2008 to 2010) using same transect and time. Observations were carried out with the aid of $8 \times 40$ binoculars, and field characteristics were noted down on special ornithological data sheet which included species, number of individuals, activity, microhabitat, threats with other minor details. The birds were identified with the help of Ali and Ripley [10], Ali [11], and Grimmett et al. [12]. Birds sighted during our survey were categorized based on their migratory nature including resident breeder $(\mathrm{RB})$, resident $(\mathrm{R})$, and migrant $(\mathrm{M})$ according to Ali [11] and also categorized into their trophic guilds like insectivore, nectarivore, omnivore, scavenger, frugivore, carnivore, piscivore, and granivore according to Willis [13], Robinson et al. [14], and Anjos [15].

2.3. Data Analysis. The data collected during the whole study period were analyzed in PAST statistical software [16] to calculate species diversity, density, richness, and species composition by using the following formulae.

(A) Shannon Wiener diversity index $\left(H^{\prime}\right) H^{\prime}=\sum P_{i} \times$ $\ln \left(P_{i}\right)$, where

$$
\begin{aligned}
& H=\text { index of species diversity, } \\
& P_{i}=\text { proportion of total sample belonging to the } \\
& i \text { th species, } \\
& \ln =\text { natural logl. }
\end{aligned}
$$

(B) Density $=$ no. of individuals/total area surveyed (in hectare). 


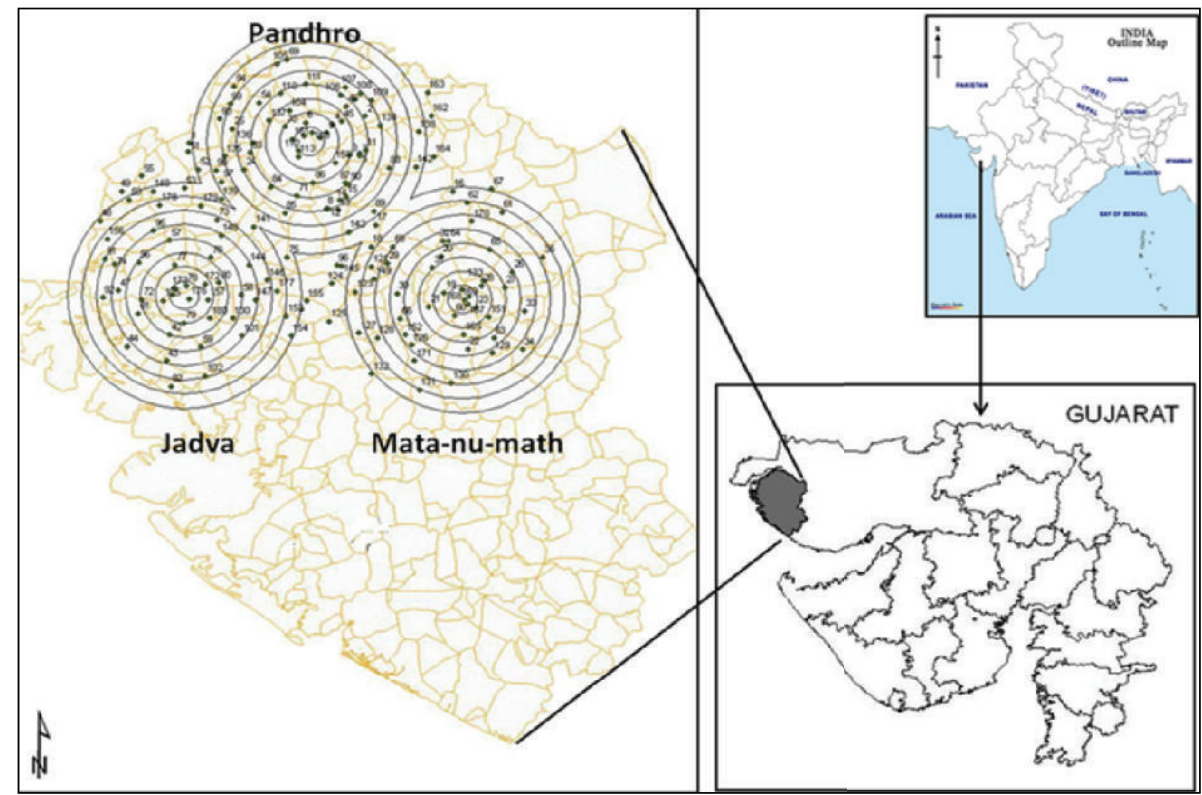

FIGURE 1: Location of the study area and transects (spots) and various zones (in circles) made around the three selected mines (Pandhro, Mata-na-madh, and Jadva) of western Kachchh for bird study.

(C) Menhinick richness index $=$ the ratio of the number of taxa to the square root of sample size.

(D) Buzas and Gibson's evenness: $e^{H} / S$.

The rarefaction analysis was carried by using Biodiversity Pro software, 1997, to cope up the problem in comparing diversity among various land cover or habitat categories evaluated during the present study.

2.4. Hypothesis Testing. The null hypotheses tested for significance of differences was the following.

Ha: There is no impact of mining on the distribution and diversity of bird species.

Analysis of variance (ANOVA) was used to test the hypotheses, and Tukey's HSD post hoc analysis was carried to identify specific variables that differ significantly. The variables used for testing the hypotheses included the number of species, density, Shannon diversity index, and Menhinick species richness index. These variables representing bird species diversity were evaluated from each mine zone from $\mathrm{Z1}$ to $\mathrm{Z9}$.

Further, cluster analysis was made to quantify the resemblance among bird communities inhabiting various mine zones. Cluster analysis was performed on bird species. Abundance data were obtained from transect survey using minimum variances technique, known as Ward's method, which has been recognized as the best way to classify the ecological communities and to identify community structure. The cluster analysis generated a dendrogram providing hierarchically nested groups or clusters representing distinct bird communities, which were represented by subclusters.

\section{Results}

On surveying the mining and surrounding peripheral areas of the study area including all kinds of habitat, 252 species of birds (see Table 2) were recorded in western Kachchh. Various zones from zone 1 to zone 9 made for this study were located in and around the mines as shown in Figure 1. Zone 1 and zone 2 are overlapping with mines and are very close to the mines while zone 9 is the farthest from the mines. These zones were described as Z1, Z2, Z3, Z4, Z5, Z6, Z7, Z8, and Z9. The total number of bird species, mean number of species/transects, and their density recorded in various zones are shown in Table 1. Among these zones, $\mathrm{Z} 1$ recorded the least number of species and Z5 recorded the highest number of species of birds, while mean number of species/transects recorded its minimum value in $\mathrm{Z} 2$ and its maximum in $\mathrm{Z7}$. Among the zones, Larks were the most abundant group of birds in Z1, Z3, and Z9, common coot was the most abundant in $\mathrm{Z} 2, \mathrm{Z} 5$, and $\mathrm{Z} 8$, while lesser short-toed lark, common crane, and white pelican were the most abundant in Z4, Z6, and $Z 7$, respectively. The density or mean number of individ$\mathrm{ual} /$ ha recorded its highest value in Z5 (322) and its lowest in $\mathrm{Z1}$ (204) in comparison to the other zones.

The overall diversity of the birds was rich within each zone of mining areas (Figure 2) with the highest value in $\mathrm{Z8}\left(H^{\prime}=\right.$ 4.47) and the lowest in $\mathrm{Z} 1\left(H^{\prime}=4.10\right)$ and $\mathrm{Z} 4\left(H^{\prime}=4.10\right)$, while diversity of bird species/transects recorded the highest value in $\mathrm{Z9}\left(H^{\prime}=3.40\right)$ and the lowest in $\mathrm{Z2}\left(H^{\prime}=2.71\right)$. The species richness in various zones and per transect within each zone was calculated (Figure 3), and found that the Menhinick species richness index/zone was recorded the highest value in $\mathrm{Z1}$ (2.92) and the lowest in Z4 (1.18), whereas Menhinick species richness index/transect recorded the highest value in Z9 (2.65) and the lowest in Z2 (1.96). Similarly, the species 
TABLE 1: Bird species diversity and density in various zones of mining areas.

\begin{tabular}{lccc}
\hline Zone & $\begin{array}{c}\text { Total number } \\
\text { of species }\end{array}$ & $\begin{array}{c}\text { No. of } \\
\text { species/transects }\end{array}$ & $\begin{array}{c}\text { Mean } \\
\text { density/Ha }\end{array}$ \\
\hline$Z 1$ & 114 & 31.66 & 204 \\
$Z 2$ & 153 & 31.06 & 250 \\
$Z 3$ & 126 & 34.33 & 271 \\
$Z 4$ & 139 & 32.73 & 222 \\
$Z 5$ & 195 & 37.89 & 322 \\
$Z 6$ & 149 & 39.54 & 254 \\
$Z 7$ & 175 & 40.42 & 303 \\
$Z 8$ & 154 & 35 & 270 \\
$Z 9$ & 157 & 42.04 & 248 \\
\hline
\end{tabular}

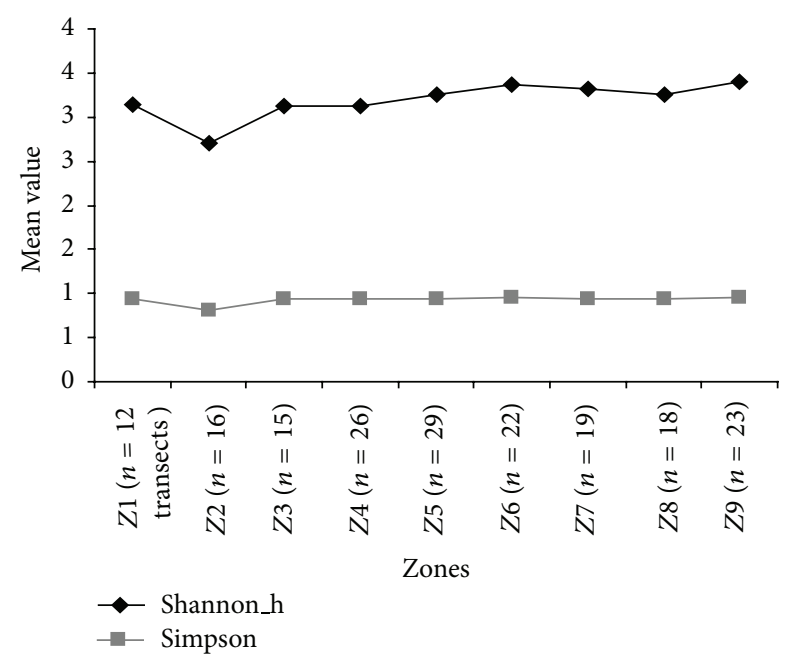

FIGURE 2: Species diversity of birds in various zones made around the mines in the study area.

evenness values of each zone and transect within were also calculated (Figure 3), and we found that the highest species evenness index was recorded in Z8 (0.57) and the lowest in Z5 (0.34), while species evenness index per transect was recorded more or less similar with the highest value in Z8 (0.78) and the lowest in $\mathrm{Z} 2$ (0.66).

On analysis of the data on various aspects of birds diversity in and around the mining areas based on various zones, it was found that diversity (Shannon diversity: $F=$ 2.984, $\mathrm{df}=8$, and $P<0.05$ ) and richness (Menhinik species richness: $F=2.403, \mathrm{df}=8$, and $P<0.05$ ) of birds in between zones differ significantly. Further, a Tukey HSD post hoc analysis results suggest that $\mathrm{Z} 2$ differs significantly from $\mathrm{Z5}$ $(P=0.018), \mathrm{Z} 6(P=0.003), \mathrm{Z7}(P=0.015), \mathrm{Z} 8(P=0.046)$, and $Z 9(P=0.001)$ in terms of mean diversity, and $Z 2$ differs significantly from Z9 $(P=0.012)$ in terms of species richness. Rarefaction analysis was done for the expected number of species in each zone of the mining areas to standardize unequal sampling sizes (Figure 4). The expected number of species recorded its highest value in Z5 and its lowest in Z1. On cluster analysis of the data, abundance of birds revealed

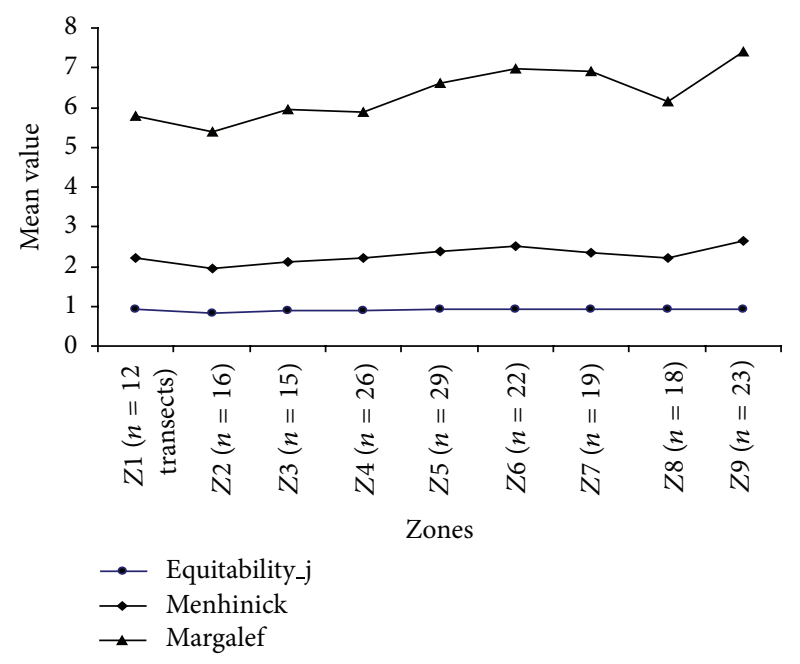

FIGURE 3: Species richness and equitability of birds in various zones made around the mines in the study area.

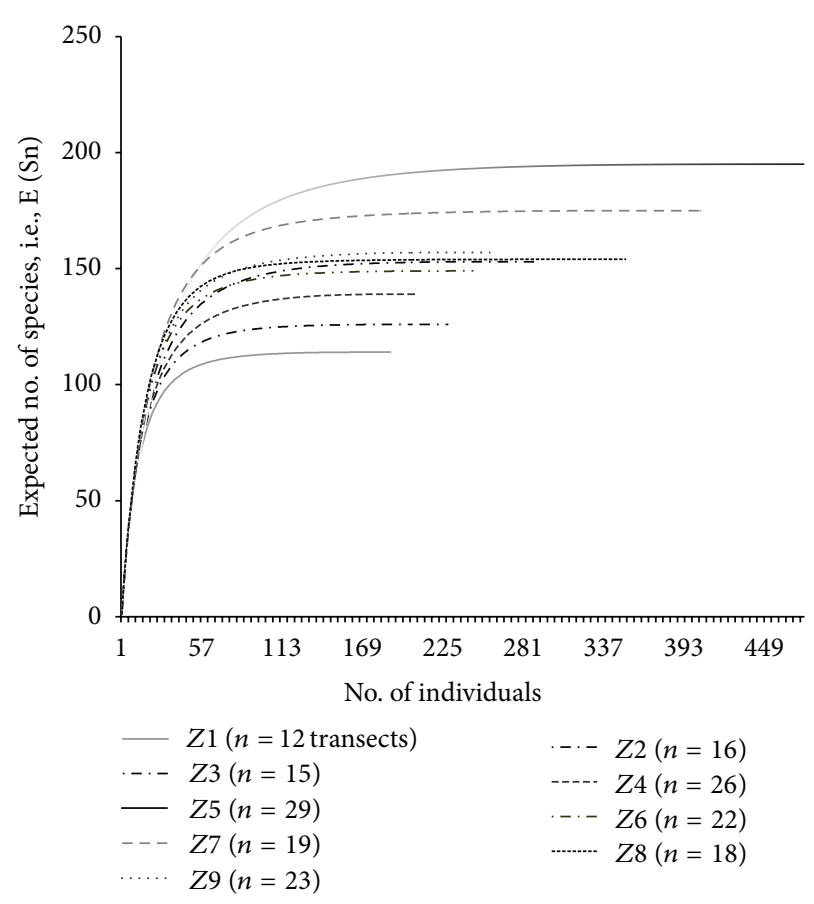

FIGURE 4: Rarefaction curve for expected number of bird species in different zones of mining environment of Kachchh, Gujarat.

great similarities among sampling points belonging to the same kind of habitat. Six distinct groups, namely, Z1, Z3; Z4, Z6; Z9; Z8; Z2; Z7, Z5 were identified (Figure 5), and we found that $Z 4$ and $Z 6$ have maximum similarity in abundance and next to that were $\mathrm{Z} 1$ and $\mathrm{Z3}$, while they have very less similarity of clusters with Z5. 
TABLE 2: Checklist of birds recorded during the study.

\begin{tabular}{|c|c|c|c|c|c|c|}
\hline Sl. no. & Order/family & Species scientific name & Common name & MS & IUCN 2010 & Feeding habit \\
\hline & Anseriformes & & & & & \\
\hline 1 & Anatidae & Anas acuta & Northern pintail & M & - & A \\
\hline 2 & & Anas clypeata & Northern shoveller & M & - & A \\
\hline 3 & & Anas penelope & Eurasian wigeon & $\mathrm{M}$ & - & A \\
\hline 4 & & Anas platyrhynchos & Mallard & $\mathrm{RM}$ & - & A \\
\hline 5 & & Anas poecilorhyncha & Spot-billed duck & $\mathrm{RM}$ & - & A \\
\hline 6 & & Anas querquedula & Garganey & M & - & A \\
\hline 7 & & Anas strepera & Gadwall & $\mathrm{M}$ & - & A \\
\hline 8 & & Aythya ferina & Common pochard & M & - & A \\
\hline 9 & & Aythya fuligula & Tufted pochard & M & - & A \\
\hline 10 & & Sarkidiornis melanotos & Comb duck & $\mathrm{R}$ & - & A \\
\hline \multirow[t]{2}{*}{11} & Dendrocygnidae & Dendrocygna javanica & Lesser whistling duck & $\mathrm{R}$ & - & A \\
\hline & Apodiiformes & & & & & \\
\hline \multirow[t]{2}{*}{12} & Apodidae & Apus affinis & House(little) swift & $\mathrm{R}$ & - & $\mathrm{I}$ \\
\hline & Ciconiiformes & & & & & \\
\hline 13 & Accipitridae & Accipiter badius & Shikra & $\mathrm{R}$ & - & $\mathrm{C}$ \\
\hline 14 & & Accipiter nisus & Eurasian sparrow-hawk & $\mathrm{R}$ & - & $\mathrm{C}$ \\
\hline 15 & & Aquila heliaca & Imperial eagle & $\mathrm{R}$ & VU & $\mathrm{C}$ \\
\hline 16 & & Aquila nipalensis & Steppe eagle & M & - & $\mathrm{C}$ \\
\hline 17 & & Aquila pomarina & Lesser spotted eagle & $\mathrm{M}$ & - & $\mathrm{C}$ \\
\hline 18 & & Aquila rapax & Tawny eagle & $\mathrm{R}$ & - & $\mathrm{C}$ \\
\hline 19 & & Butastur teesa & White-eyed buzzard & $\mathrm{R}$ & - & $\mathrm{C}$ \\
\hline 20 & & Buteo buteo & Long-legged buzzard & $\mathrm{R}$ & - & $\mathrm{C}$ \\
\hline 21 & & Circaetus gallicus & Short-toad snake eagle & $\mathrm{R}$ & - & $\mathrm{C}$ \\
\hline 22 & & Circus aeruginosus & Eurasian marsh harrier & M & - & $\mathrm{C}$ \\
\hline 23 & & Circus macrourus & Pallid harrier & M & NT & $\mathrm{C}$ \\
\hline 24 & & Circus melanoleucos & Pied harrier & $\mathrm{RM}$ & - & $\mathrm{C}$ \\
\hline 25 & & Circus pygargus & Montagu's harrier & M & - & $\mathrm{C}$ \\
\hline 26 & & Elanus caeruleus & Black-shouldered kite & $\mathrm{R}$ & - & $\mathrm{C}$ \\
\hline 27 & & Gyps bengalensis & Indian white-backed vulture & $\mathrm{R}$ & $\mathrm{CR}$ & $\mathrm{C}$ \\
\hline 28 & & Gyps indicus & Long-billed vulture & $\mathrm{R}$ & CR & $\mathrm{C}$ \\
\hline 29 & & Haliaeetus leucogaster & White-bellied sea-eagle & $\mathrm{R}$ & - & $\mathrm{C}$ \\
\hline 30 & & Haliaeetus leucoryphus & Pallas's fish-eagle & $\mathrm{RM}$ & VU & $\mathrm{C}$ \\
\hline 31 & & Haliastur Indus & Brahminy kite & $\mathrm{R}$ & - & $\mathrm{C}$ \\
\hline 32 & & Hieraaetus fasciatus & Bonelli's eagle & M & - & $\mathrm{C}$ \\
\hline 33 & & Hieraaetus pennatus & Booted eagle & $\mathrm{R}$ & - & $\mathrm{C}$ \\
\hline 34 & & Milvus migrans & Black kite & $\mathrm{R}$ & - & $\mathrm{C}$ \\
\hline 35 & & Pernis ptilorhynchus & Oriental honey-buzzard & $\mathrm{R}$ & - & $\mathrm{C}$ \\
\hline 36 & & Spilornis cheela & Crested serpent eagle & $\mathrm{R}$ & - & $\mathrm{C}$ \\
\hline 37 & Ardeidae & Ardea alba & Great egret & $\mathrm{RM}$ & - & $\mathrm{A}$ \\
\hline 38 & & Ardea cinerea & Grey heron & $\mathrm{RM}$ & - & A \\
\hline 39 & & Ardea purpurea & Purple heron & $\mathrm{RM}$ & - & A \\
\hline 40 & & Ardeola grayii & Indian pond heron & $\mathrm{R}$ & - & A \\
\hline 41 & & Bubulcus ibis & Cattle egret & $\mathrm{R}$ & - & I \\
\hline 42 & & Butorides striatus & Little green heron & $\mathrm{RM}$ & - & A \\
\hline 43 & & Egretta garzetta & Little egret & $\mathrm{R}$ & - & A \\
\hline 44 & & Egretta gularis & Western reef-egret & $\mathrm{R}$ & - & A \\
\hline 45 & & Mesophoyx intermedia & Intermediate egret & $\mathrm{R}$ & - & A \\
\hline 46 & Burhinidae & Burhinus oedicnemus & Eurasian thick-knee & $\mathrm{R}$ & - & A \\
\hline
\end{tabular}


TABLe 2: Continued.

\begin{tabular}{|c|c|c|c|c|c|c|}
\hline Sl. no. & Order/family & Species scientific name & Common name & MS & IUCN 2010 & Feeding habit \\
\hline 47 & Charadriidae & Calidris alpine & Dunlin & $\mathrm{M}$ & - & $\mathrm{A}$ \\
\hline 48 & & Charadrius alexandrinus & Kentish plover & $\mathrm{RM}$ & - & A \\
\hline 49 & & Charadrius mongolus & Lesser sand plover & $\mathrm{RM}$ & - & $\mathrm{A}$ \\
\hline 50 & & Charadrius dubius & Common ring plover & $\mathrm{RM}$ & - & A \\
\hline 51 & & Esacus magnirostris & Greater sand-plover & $\mathrm{R}$ & - & A \\
\hline 52 & & Himantopus himantopus & Black-winged stilt & $\mathrm{R}$ & - & A \\
\hline 53 & & Pluvialis squatarola & Grey plover & $\mathrm{M}$ & - & A \\
\hline 54 & & Rostratula benghalensis & Painted snipe & $\mathrm{R}$ & - & $\mathrm{A}$ \\
\hline 55 & & Tringa erythropus & Spotted redshank & M & - & $\mathrm{A}$ \\
\hline 56 & & Vanellus indicus & Red-wattled lapwing & $\mathrm{R}$ & - & I \\
\hline 57 & & Vanellus malabaricus & Yellow-wattled lapwing & $\mathrm{R}$ & - & I \\
\hline 58 & & Calidris minuta & Little stint & $\mathrm{M}$ & - & A \\
\hline 59 & Ciconiidae & Ephippiorhynchus asiaticus & Black necked stork & $\mathrm{R}$ & NT & $\mathrm{P}$ \\
\hline 60 & & Mycteria leucocephala & Painted stork & $\mathrm{RM}$ & NT & A \\
\hline 61 & Falconidae & Falco chicquera & Red-necked falcon & $\mathrm{R}$ & - & $\mathrm{C}$ \\
\hline 62 & & Falco jugger & Lagger falcon & $\mathrm{R}$ & NT & $\mathrm{C}$ \\
\hline 63 & & Falco naumanni & Lesser kestrel & $\mathrm{R}$ & VU & $\mathrm{C}$ \\
\hline 64 & & Falco subbuteo centralasiae & Eurasian hobby & $\mathrm{M}$ & - & $\mathrm{C}$ \\
\hline 65 & & Falco tinnunculus & Common kestrel & $\mathrm{RM}$ & - & $\mathrm{C}$ \\
\hline 66 & Glareolidae & Cursorius coromandelicus & Indian courser & $\mathrm{R}$ & - & I \\
\hline 67 & Laridae & Calidris alba & Sanderling & $\mathrm{RM}$ & - & $\mathrm{P}$ \\
\hline 68 & & Chlidonias leucopterus & White winged black tern & $\mathrm{RM}$ & - & $\mathrm{P}$ \\
\hline 69 & & Larus brunnicephalus & Brown-headed gull & $\mathrm{RM}$ & - & $\mathrm{P}$ \\
\hline 70 & & Larus cachinnans & Yellow-legged gull & $\mathrm{M}$ & - & $\mathrm{P}$ \\
\hline 71 & & Larus fuscus & Lesser black backed gull & $\mathrm{M}$ & - & $\mathrm{P}$ \\
\hline 72 & & Sterna acuticauda & Black-bellied tern & $\mathrm{M}$ & NT & $\mathrm{P}$ \\
\hline 73 & & Sterna aurantia & River tern & $\mathrm{R}$ & - & A \\
\hline 74 & & Sterna caspia & Caspian tern & $\mathrm{RM}$ & - & $\mathrm{P}$ \\
\hline 75 & Pelecanidae & Pelecanus onocrotalus & Great white-pelican & $\mathrm{RM}$ & - & $\mathrm{P}$ \\
\hline 76 & Phalacrocoracidae & Phalacrocorax carbo & Great cormorant & $\mathrm{R}$ & - & $\mathrm{A}$ \\
\hline 77 & & Phalacrocorax fuscicollis & Indian cormorant & $\mathrm{R}$ & - & A \\
\hline 78 & & Phalacrocorax niger & Little cormorant & $\mathrm{R}$ & - & $\mathrm{A}$ \\
\hline 79 & Phoenicopteridae & Phoenicopterus minor & Lesser flamingo & $\mathrm{RM}$ & NT & A \\
\hline 80 & & Phoenicopterus ruber & Greater flamingo & $\mathrm{RM}$ & - & A \\
\hline 81 & Podicipedidae & Podiceps cristatus & Great crested grebe & $\mathrm{RM}$ & - & $\mathrm{A}$ \\
\hline 82 & & Podiceps nigricollis & Black-necked grebe & $\mathrm{M}$ & - & A \\
\hline 83 & & Tachybaptus ruficollis & Little grebe & $\mathrm{R}$ & - & $\mathrm{A}$ \\
\hline 84 & Pteroclididae & Pterocles alchata & White-bellied sandgrouse & $\mathrm{M}$ & - & G \\
\hline 85 & & Pterocles exustus & Chestnut-bellied sandgrouse & $\mathrm{R}$ & - & G \\
\hline 86 & & Pterocles indicus & Painted sandgrouse & $\mathrm{R}$ & - & G \\
\hline 87 & Scolopacidae & Actitis hypoleucos & Common sandpiper & $\mathrm{R}$ & - & A \\
\hline 88 & & Limosa limosa & Black-tailed godwit & $\mathrm{M}$ & NT & $\mathrm{A}$ \\
\hline 89 & & Numenius phaeopus & Whimbrel & $\mathrm{M}$ & - & $\mathrm{A}$ \\
\hline 90 & & Tringa glareola & Wood sandpiper & $\mathrm{M}$ & - & $\mathrm{A}$ \\
\hline 91 & & Tringa nebularia & Common greenshank & $\mathrm{M}$ & - & $\mathrm{A}$ \\
\hline 92 & & Tringa ochropus & Green sandpiper & $\mathrm{M}$ & - & A \\
\hline 93 & & Tringa stagnatilis & Marsh sandpiper & $\mathrm{M}$ & - & $\mathrm{A}$ \\
\hline 94 & Threskiornithidae & Platalea leucorodia & Eurasian spoonbill & $\mathrm{RM}$ & - & A \\
\hline 95 & & Plegadis falcinellus & Glossy ibis & $\mathrm{RM}$ & - & I \\
\hline 96 & & Pseudibis papillosa & Black ibis & $\mathrm{R}$ & - & I \\
\hline 97 & & Threskiornis melanocephalus & Black-headed ibis & $\mathrm{RM}$ & NT & A \\
\hline
\end{tabular}


TABle 2: Continued.

\begin{tabular}{|c|c|c|c|c|c|c|}
\hline Sl. no. & Order/family & Species scientific name & Common name & MS & IUCN 2010 & Feeding habit \\
\hline & Columbiformes & & & & & \\
\hline 98 & Columbidae & Columba livia & Rock pigeon & $\mathrm{R}$ & - & G \\
\hline 99 & & Streptopelia chinensis & Spotted dove & $\mathrm{R}$ & - & G \\
\hline 100 & & Streptopelia decaocto & Eurasian collared dove & $\mathrm{R}$ & - & G \\
\hline 101 & & Streptopelia orientalis & Oriental turtle-dove & $\mathrm{RM}$ & - & G \\
\hline 102 & & Streptopelia senegalensis & Laughing dove & $\mathrm{R}$ & - & G \\
\hline \multirow[t]{2}{*}{103} & & Streptopelia tranquebarica & Red-collared dove & $\mathrm{R}$ & - & G \\
\hline & Coraciiformes & & & & & \\
\hline 104 & Alcedinidae & Alcedo Hercules & Common kingfisher & $\mathrm{R}$ & - & $\mathrm{P}$ \\
\hline 105 & Cerylidae & Ceryle rudis & Lesser pied kingfisher & $\mathrm{R}$ & - & $\mathrm{P}$ \\
\hline 106 & Coraciidae & Coracias benghalensis & Indian roller & $\mathrm{R}$ & - & I \\
\hline 107 & & Coracias garrulus & European roller & $\mathrm{RM}$ & NT & I \\
\hline 108 & Dacelonidae & Halcyon smyrnensis & White-throated & $\mathrm{R}$ & - & $\mathrm{P}$ \\
\hline 109 & Meropidae & Merops leschenaulti & Chestnut-headed bee-eater & $\mathrm{R}$ & - & I \\
\hline 110 & & Merops orientalis & Green bee-eater & $\mathrm{R}$ & - & I \\
\hline 111 & & Merops persicus & Blue-cheeked bee-eater & $\mathrm{RM}$ & - & I \\
\hline \multirow[t]{2}{*}{112} & & Merops philippinus & Blue-tailed bee-eater & $\mathrm{RM}$ & - & $\mathrm{I}$ \\
\hline & Cuculiformes & & & & & \\
\hline 113 & Centropodidae & Centropus sinensis & Greater coucal & $\mathrm{R}$ & - & $\mathrm{O}$ \\
\hline 114 & Cuculidae & Cuculus canorus & Pied-crested cuckoo & $\mathrm{R}$ & - & I \\
\hline 115 & & Eudynamys scolopacea & Asian koel & $\mathrm{R}$ & - & $\mathrm{F}$ \\
\hline \multirow[t]{2}{*}{116} & & Phaenicophaeus leschenaultii & Sirkeer cuckoo & $\mathrm{R}$ & - & $\mathrm{O}$ \\
\hline & Galliformes & & & & & \\
\hline 117 & Phasianidae & Coturnix coromandelica & Rain quail & $\mathrm{R}$ & - & G \\
\hline 118 & & Coturnix coturnix & Common quail & $\mathrm{R}$ & - & G \\
\hline 119 & & Francolinus francolinus & Black francolin & $\mathrm{R}$ & - & G \\
\hline 120 & & Francolinus pictus & Painted francolin & $\mathrm{R}$ & - & G \\
\hline 121 & & Francolinus pondicerianus & Grey francolin & $\mathrm{R}$ & - & G \\
\hline 122 & & Pavo cristatus & Indian peafowl & $\mathrm{R}$ & - & G \\
\hline \multirow[t]{2}{*}{123} & & Perdicula asiatica & Jungle bush quail & $\mathrm{R}$ & - & G \\
\hline & Gruiformes & & & & & \\
\hline 124 & Gruidae & Grus grus & Common crane & M & - & $\mathrm{O}$ \\
\hline 125 & & Grus virgo & Demoiselle crane & $\mathrm{M}$ & - & $\mathrm{O}$ \\
\hline 126 & Otididae & Ardeotis nigriceps & Great indian bustard & $\mathrm{R}$ & $\mathrm{EN}$ & G \\
\hline 127 & Rallidae & Fulica atra & Common coot & $\mathrm{R}$ & - & A \\
\hline \multirow[t]{2}{*}{128} & & Gallinula chloropus & Common moorhen & $\mathrm{R}$ & - & A \\
\hline & Passeriformes & & & & & \\
\hline 129 & Alaudidae & Alauda arvensis & Eurasian skylark & $\mathrm{R}$ & - & G \\
\hline 130 & & Alauda gulgula & Oriental skylark & $\mathrm{R}$ & - & G \\
\hline 131 & & Ammomanes phoenicurus & Rufous-tailed lark & $\mathrm{R}$ & - & G \\
\hline 132 & & Calandrella brachydactyla & Greater short-toed lark & M & - & G \\
\hline 133 & & Calandrella rufescens & Lesser short-toed lark & $\mathrm{RM}$ & - & G \\
\hline 134 & & Eremopterix grisea & Ashy-crowned sparrow-lark & $\mathrm{R}$ & - & G \\
\hline 135 & & Galerida cristata & Crested lark & $\mathrm{R}$ & - & G \\
\hline 136 & & Galerida deva & Sykes's crested lark & $\mathrm{R}$ & - & G \\
\hline 137 & & Galerida malabarica & Malabar lark & $\mathrm{RM}$ & - & G \\
\hline 138 & & Mirafra affinis & Jerdon's bushlark & $\mathrm{RM}$ & - & G \\
\hline 139 & & Mirafra cantillans & Singing bushlark & $\mathrm{R}$ & - & G \\
\hline 140 & & Mirafra erythroptera & Indian bushlark & $\mathrm{R}$ & - & G \\
\hline 141 & & Mirafra erythroptera & Red-winged bush-lark & $\mathrm{R}$ & - & G \\
\hline
\end{tabular}


TABLE 2: Continued.

\begin{tabular}{|c|c|c|c|c|c|c|}
\hline Sl. no. & Order/family & Species scientific name & Common name & MS & IUCN 2010 & Feeding habit \\
\hline 142 & Certhiidae & Salpornis spilonotus & Spotted creeper & $\mathrm{R}$ & - & I \\
\hline 143 & Cisticolidae & Orthotomus sutorius & Tailor bird & $\mathrm{R}$ & - & I \\
\hline 144 & & Prinia buchanani & Rufous-fronted prinia & $\mathrm{R}$ & - & I \\
\hline 145 & & Prinia hodgsonii & Grey-breasted prinia & $\mathrm{R}$ & - & I \\
\hline 146 & & Prinia inornata & Plain prinia & $\mathrm{R}$ & - & I \\
\hline 147 & & Prinia socialis & Ashy prinia & $\mathrm{R}$ & - & I \\
\hline 148 & & Prinia sylvatica & Jungle prinia & $\mathrm{R}$ & - & I \\
\hline 149 & Corvidae & Aegithina nigrolutea & Marshall's iora & $\mathrm{R}$ & - & I \\
\hline 150 & & Coracina macei & Large cuckoo shrike & $\mathrm{R}$ & - & I \\
\hline 151 & & Corvus splendens & House crow & $\mathrm{R}$ & - & $\mathrm{O}$ \\
\hline 152 & & Dendrocitta vagabunda & Rufous treepie & $\mathrm{R}$ & - & I \\
\hline 153 & & Dicrurus caerulescens & White-bellied drongo & $\mathrm{R}$ & - & I \\
\hline 154 & & Dicrurus macrocercus & Black drongo & $\mathrm{R}$ & - & I \\
\hline 155 & & Pericrocotus cinnamomeus & Small minivet & $\mathrm{R}$ & - & I \\
\hline 156 & & Rhipidura aureola & White-browed fantail & $\mathrm{R}$ & - & I \\
\hline 157 & & Tephrodornis gularis & Large wood shrike & $\mathrm{R}$ & - & I \\
\hline 158 & & Tephrodornis pondicerianus & Common woodshrike & $\mathrm{R}$ & - & I \\
\hline 159 & Fringillidae & Emberiza buchanani & Grey-necked bunting & M & - & G \\
\hline 160 & & Emberiza cia & Rock bunting & $\mathrm{M}$ & - & G \\
\hline 161 & & Emberiza melanocephala & Ortolan bunting & $\mathrm{M}$ & - & G \\
\hline 162 & & Emberiza striolata & House bunting & $\mathrm{R}$ & - & G \\
\hline 163 & & Melophus lathami & Crested bunting & $\mathrm{R}$ & - & G \\
\hline 164 & Hirundinidae & Hirundo concolor & Dusky crag-martin & $\mathrm{R}$ & - & I \\
\hline 165 & & Hirundo daurica & Red-rump swallow & $\mathrm{R}$ & - & I \\
\hline 166 & & Hirundo fluvicola & Streak-throated swallow & $\mathrm{R}$ & - & I \\
\hline 167 & & Hirundo rupestris & Eurasian crag-martin & $\mathrm{R}$ & - & I \\
\hline 168 & & Hirundo rustica & Barn swallow & $\mathrm{RM}$ & - & I \\
\hline 169 & & Hirundo smithii & Wire-tailed swallow & $\mathrm{R}$ & - & I \\
\hline 170 & Laniidae & Lanius collurio & Red-backed shrike & $\mathrm{R}$ & - & $\mathrm{C}$ \\
\hline 171 & & Lanius cristatus & Brown shrike & $\mathrm{M}$ & - & $\mathrm{C}$ \\
\hline 172 & & Lanius isabellinus & Rufous-tailed shrike & $\mathrm{RM}$ & - & $\mathrm{C}$ \\
\hline 173 & & Lanius meridionalis & Southern grey shrike & $\mathrm{R}$ & - & $\mathrm{C}$ \\
\hline 174 & & Lanius schach & Long-tailed shrike & $\mathrm{R}$ & - & $\mathrm{C}$ \\
\hline 175 & & Lanius schach canipes & Rufous-backed shrike & $\mathrm{R}$ & - & $\mathrm{C}$ \\
\hline 176 & & Lanius vittatus & Bay-backed shrike & $\mathrm{R}$ & - & $\mathrm{C}$ \\
\hline 177 & Muscicapidae & Cercomela fusca & Brown rock chat & $\mathrm{R}$ & - & I \\
\hline 178 & & Cercotrichas galactotes & Rufous chat & $\mathrm{M}$ & - & I \\
\hline 179 & & Copsychus saularis & Oriental magpie robin & $\mathrm{R}$ & - & I \\
\hline 180 & & Culicicapa ceylonensis & Grey-headed flycatcher & $\mathrm{R}$ & - & I \\
\hline 181 & & Ficedula parva & Red-throated flycatcher & $\mathrm{R}$ & - & I \\
\hline 182 & & Ficedula superciliaris & Ultramarine flycatcher & $\mathrm{R}$ & - & I \\
\hline 183 & & Muscicapa striata & Spotted flycatcher & $\mathrm{RM}$ & - & I \\
\hline 184 & & Oenanthe deserti & Desert wheatear & $\mathrm{RM}$ & - & I \\
\hline 185 & & Oenanthe isabellina & Isabelline wheatear & $\mathrm{RM}$ & - & I \\
\hline 186 & & Oenanthe picata & Variable wheatear & $\mathrm{M}$ & - & I \\
\hline 187 & & Phoenicurus erythronotus & Rufous-backed redstart & $\mathrm{RM}$ & - & I \\
\hline 188 & & Phoenicurus ochruros & Black redstart & $\mathrm{RM}$ & - & I \\
\hline 189 & & Saxicola caprata & Pied bush chat & $\mathrm{R}$ & - & I \\
\hline 190 & & Saxicola jerdoni & Pied chat & $\mathrm{R}$ & - & I \\
\hline 191 & & Saxicola macrorhyncha & Stoliczka’s bushchat & $\mathrm{RM}$ & VU & $\mathrm{I}$ \\
\hline
\end{tabular}


TABLe 2: Continued.

\begin{tabular}{|c|c|c|c|c|c|c|}
\hline Sl. no. & Order/family & Species scientific name & Common name & MS & IUCN 2010 & Feeding habit \\
\hline 192 & & Saxicola torquata & Common stonechat & $\mathrm{RM}$ & - & I \\
\hline 193 & & Saxicoloides fulicata & Indian robin & $\mathrm{R}$ & - & I \\
\hline 194 & & Turdus naumanni & Dusky thrush & $\mathrm{RM}$ & - & I \\
\hline 195 & & Turdus obscurus & Eyebrowed thrush & $\mathrm{RM}$ & - & I \\
\hline 196 & & Zoothera citrina & Orange-headed thrush & $\mathrm{R}$ & - & I \\
\hline 197 & Nectariniidae & Aethopyga siparaja & Crimson sunbird & $\mathrm{R}$ & - & $\mathrm{N}$ \\
\hline 198 & & Dicaeum agile & Thick-billed flowerpecker & $\mathrm{R}$ & - & $\mathrm{N}$ \\
\hline 199 & & Nectarinia asiatica & Purple sunbird & $\mathrm{R}$ & - & $\mathrm{N}$ \\
\hline 200 & & Nectarinia zeylonica & Purple-rumped sunbird & $\mathrm{R}$ & - & $\mathrm{N}$ \\
\hline 201 & Paridae & Parus major & Great tit & $\mathrm{R}$ & - & I \\
\hline 202 & & Parus nuchalis & Pied tit & $\mathrm{R}$ & VU & I \\
\hline 203 & Passeridae & Anthus campestris & Tawny pipit & $\mathrm{RM}$ & - & G \\
\hline 204 & & Anthus godlewski & Blyth's pipit & $\mathrm{RM}$ & - & G \\
\hline 205 & & Anthus rufulus & Paddyfield pipit & $\mathrm{R}$ & - & G \\
\hline 206 & & Anthus similis jerdoni & Brown rock pipit & M & - & G \\
\hline 207 & & Anthus trivialis & Tree pipit & $\mathrm{RM}$ & - & G \\
\hline 208 & & Dendronanthus indicus & Forest wagtail & $\mathrm{RM}$ & - & I \\
\hline 209 & & Lonchura malabarica & Indian silverbill & $\mathrm{R}$ & - & G \\
\hline 210 & & Lonchura striata & White-rumped munia & $\mathrm{R}$ & - & G \\
\hline 211 & & Motacilla alba & White wagtail & $\mathrm{RM}$ & - & I \\
\hline 212 & & Motacilla cinerea & Grey wagtail & $\mathrm{RM}$ & & I \\
\hline 213 & & Motacilla citreola & Citrine wagtail & $\mathrm{RM}$ & - & I \\
\hline 214 & & Motacilla flava & Yellow wagtail & $\mathrm{RM}$ & - & I \\
\hline 215 & & Passer domesticus & House sparrow & $\mathrm{R}$ & - & G \\
\hline 216 & & Petronia xanthocollis & Chestnut-shouldered petronia & $\mathrm{R}$ & - & G \\
\hline 217 & & Ploceus philippinus & Baya weaver & $\mathrm{R}$ & - & G \\
\hline 218 & Pycnonotidae & Pycnonotus cafer & Red-vented bulbul & $\mathrm{R}$ & - & I \\
\hline 219 & & Pycnonotus leucotis & White-eared bulbul & $\mathrm{R}$ & - & I \\
\hline 220 & Sturnidae & Acridotheres ginginianus & Bank myna & $\mathrm{R}$ & - & $\mathrm{O}$ \\
\hline 221 & & Acridotheres tristis & Common myna & $\mathrm{R}$ & - & I \\
\hline 222 & & Sturnus pagodarum & Brahminy starling & $\mathrm{R}$ & - & $\mathrm{O}$ \\
\hline 223 & & Sturnus roseus & Rosy starling & $\mathrm{M}$ & - & G \\
\hline 224 & Sylviidae & Acrocephalus aedon & Thick-billed warbler & $\mathrm{M}$ & - & I \\
\hline 225 & & Acrocephalus dumetorum & Blyth's reed-warbler & $\mathrm{RM}$ & - & I \\
\hline 226 & & Acrocephalus stentoreus & Indian great reed-warbler & $\mathrm{RM}$ & - & I \\
\hline 227 & & Chaetornis striatus & Bristled grass-warbler & $\mathrm{M}$ & VU & I \\
\hline 228 & & Chrysomma sinense & Yellow-eyed babbler & $\mathrm{R}$ & - & I \\
\hline 229 & & Cisticola juncidis & Streak fantail warbler & $\mathrm{R}$ & - & I \\
\hline 230 & & Hippolais caligata & Booted warbler & $\mathrm{RM}$ & - & I \\
\hline 231 & & Locustella naevia & Grasshopper warbler & $\mathrm{R}$ & - & I \\
\hline 232 & & Phylloscopus inornatus & Desert warbler & $\mathrm{R}$ & - & $\mathrm{F}$ \\
\hline 233 & & Phylloscopus magnirostris & Large-billed leaf-warbler & $\mathrm{M}$ & - & I \\
\hline 234 & & Phylloscopus neglectus & Plain-leaf warbler & $\mathrm{R}$ & - & $\mathrm{F}$ \\
\hline 235 & & Phylloscopus trochiloides & Greenish leaf-warbler & $\mathrm{M}$ & - & I \\
\hline 236 & & Sylvia communis & Greater whitethroat & $\mathrm{R}$ & - & I \\
\hline 237 & & Sylvia curruca & Lesser whitethroat & $\mathrm{M}$ & - & I \\
\hline 238 & & Sylvia hortensis & Orphean warbler & $\mathrm{M}$ & - & I \\
\hline 239 & & Turdoides caudatus & Common babbler & $\mathrm{R}$ & - & G \\
\hline 240 & & Turdoides malcolmi & Large grey babbler & $\mathrm{R}$ & - & G \\
\hline 241 & & Turdoides striatus & Jungle babbler & $\mathrm{R}$ & - & G \\
\hline 242 & Zosteropidae & Zosterops palpebrosus & Oriental white-eye & $\mathrm{RM}$ & - & $\mathrm{F}$ \\
\hline
\end{tabular}


TABLE 2: Continued.

\begin{tabular}{|c|c|c|c|c|c|c|}
\hline Sl. no. & Order/family & Species scientific name & Common name & MS & IUCN 2010 & Feeding habit \\
\hline & Piciformes & & & & & \\
\hline 243 & Picidae & Dendrocopos mahrattensis & Yellow-fronted pied woodpecker & $\mathrm{R}$ & - & I \\
\hline \multirow[t]{2}{*}{244} & & Jynx torquilla & Eurasian wryneck & $\mathrm{R}$ & - & I \\
\hline & Psittaciformes & & & & & \\
\hline 245 & Psittacidae & Psittacula cyanocephala & Plum-headed parakeet & $\mathrm{R}$ & - & $\mathrm{F}$ \\
\hline \multirow[t]{2}{*}{246} & & Psittacula krameri & Rose-ringed parakeet & $\mathrm{R}$ & - & $\mathrm{F}$ \\
\hline & Strigiformes & & & & & \\
\hline 247 & Strigidae & Bubo bubo & Eurasian eagle owl & $\mathrm{R}$ & - & $\mathrm{C}$ \\
\hline 248 & & Otus bakkamoena & Collared scops-owl & $\mathrm{R}$ & - & I \\
\hline 249 & Caprimulgidae & Caprimulgus asiaticus & Indian nightjar & $\mathrm{R}$ & - & I \\
\hline 250 & & Caprimulgus europaeus & Eurasian nightjar & $\mathrm{M}$ & - & I \\
\hline \multirow[t]{2}{*}{251} & Strigidae & Athene brama & Spotted owlet & $\mathrm{R}$ & - & $\mathrm{C}$ \\
\hline & Upupiformes & & & & & \\
\hline 252 & Upupidae & Uрира ерорs & Common hoopoe & $\mathrm{RM}$ & - & I \\
\hline
\end{tabular}

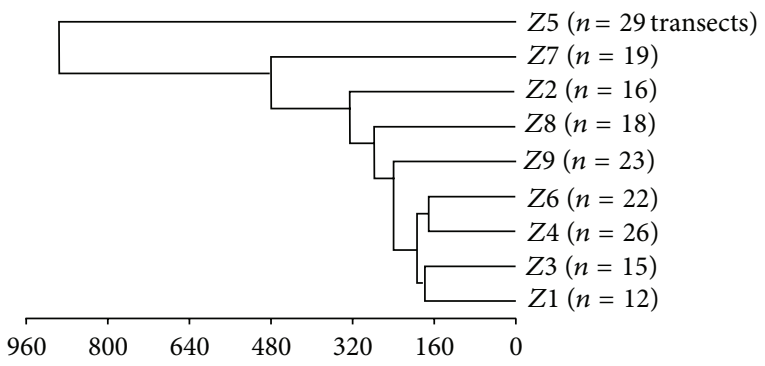

FIGURE 5: Clusters of zones based on abundance of bird species in mining areas of Kachchh, Gujarat.

\section{Discussion}

Ecosystem disturbance is one of the major phenomena in recent times which alters the relationship of organisms and their habitat in time and space. The extraction of mineral resources through mining activities in wildlife areas is one of the major factors for ecosystem disturbances and habitat fragmentation results impact on the survival of precious wildlife. Large-scale denudation of forest cover, scarcity of water, pollution of air, water, and soil, and degradation of agricultural lands are some of the conspicuous environmental implications of mining in western Kachchh. The result of the study reveals that the whole mining areas and its surrounding areas are rich in terms of avifaunal diversity and abundance. It's also found that the species diversity, richness, and abundance were less in the zones which are close to the mines within $4 \mathrm{~km}$ radius to mines. Interestingly, the species diversity was found lowest in zone 2 in comparison to zone 1 which is probably due to the frequency of mining activities which were more in zone 2 in comparison to zone 1 . The mining activity is completed in zone 1 and as a result some aquatic water bodies were developed in this zone which attracts aquatic birds to it. Salovarov and Kuznetsova [17] also found similar type of results in coal mining areas of Angara region, Russia. At the present time, mining activities are more frequent in zone 2 as a result of bird species diversity, and abundance recorded less in comparison to the other zones. The species diversity, abundance, and richness were found highest in zones beyond zone 5 to zone 9 which were located in between $8 \mathrm{~km}$ to $18 \mathrm{~km}$ from the mines. The expected number of species in zone 5 was found to be the highest and zone 2 was the lowest. Some of the species of birds may adapt to the human habitation and mining environment as it provides easy food and habitat. Smith et al. [18] found that mining has no impact on birds, but it helps in supporting some breeding birds. The species of birds which naturally survive in certain kind of habitat in this area will get more threat from the mining activities as the mining in this areas is opencast type.

\section{Conclusion}

These results indicate that mining and its associated activities have some impacts on the diversity and distribution of birds in Kachchh region of Gujarat. The enhancement of mining and other developmental activities after the earthquake and in recent times has worsened the situation which will further increase the rate of habitat destruction and their avifaunal diversity. Apart from the above, western Kachchh is the gateway of large number of migratory birds to India which will also be affected if the rate of mining activities and industrial development will go on like today.

\section{Acknowledgments}

The authors are grateful to the, Director of the Gujarat Institute of Desert Ecology, Bhuj, for his support and for providing facility. The authors are also grateful to scientists and researchers of the Terrestrial Ecology Division, GUIDE, for their help and encouragement. 


\section{References}

[1] C. D. Lester, The Birds of Kutch, Kutch Darba, Bhuj, Kutch, 1904.

[2] S. Ali, The Birds of Kutch, Oxford University Press, Bombay, India, 1st edition, 1945.

[3] S. D. Ripley, "Review: the birds of Kutch," The Auk, vol. 65, no. 1, p. 148, 1948.

[4] M. K. Himmatsinhji, "More bird notes from Kutch," Journal of the Bombay Natural History Society, vol. 55, no. 3, pp. 575-576, 1959.

[5] K. Maharao, "Some bird records from Kutch," Journal of the Bombay Natural History Society, vol. 65, no. 1, p. 225, 1968.

[6] J. K. Tiwari, "Checklist of birds of Kachchh," 2011, http://www .kolkatabirds.com/gujarat/gujaratclist.htm.

[7] S. K. Sen, "Birds of Kachchh, Gujarat," 2012, http://www .kolkatabirds.com/gujarat/gujaratclist.htm.

[8] C. J. Bibby, N. D. Burgess, and D. A. Hill, Bird Census Techniques, Academic Press, London, UK, 1992.

[9] J. S. Dieni and S. L. Jones, "A field test of the area search method for measuring breeding bird populations," Journal of Field Ornithology, vol. 73, no. 3, pp. 253-257, 2002.

[10] S. Ali and S. D. Ripley, A Pictorial Guide to the Birds of the Indian Subcontinent, Bombay Natural History Society. Oxford University Press, Bombay, India, 1983.

[11] S. Ali, Book of Indian Birds, Bombay Natural History Society, 2002.

[12] R. Grimmett, C. Inskipp, and T. Inskipp, Pocket Guide To the Birds of the Indian Sub-Continent, Oxford University Press, New Delhi, India, 2006.

[13] E. O. Willis, "The composition of avian communities in reminiscent woodlots in southern Brazil," Papéis Avulsos De Zoologia, vol. 33, no. 1, pp. 1-25, 1979.

[14] S. K. Robinson, J. G. Blake, and R. O. Bierregaard Jr., "Birds of Four Neotropical Forests," in Four Neotropical Rainforest. New Haven, A. H. Fentry, Ed., pp. 237-269, Yale University Press, London, UK, 1990.

[15] L. Anjos, "Bird communities in five Atlantic forest fragments in southern Brazil," Ornithologia Neotropical, vol. 12, pp. 11-27, 2001.

[16] Ø. Hammer, D. A. T. Harper, and P. D. Ryan, "Past: paleontological statistics software package for education and data analysis," Palaeontologia Electronica, vol. 4, no. 1, 2001.

[17] V. O. Salovarov and D. V. Kuznetsova, "Impact of coal mining on bird distribution in Upper Angara Region," Izvestiia Akademii nauk. Seriia biologicheskaia, no. 2, pp. 248-251, 2006.

[18] A. C. Smith, J. A. Virgl, D. Panayi, and A. R. Armstrong, "Effects of a diamond mine on tundra-breeding birds," Arctic, vol. 58, no. 3, pp. 295-304, 2005. 

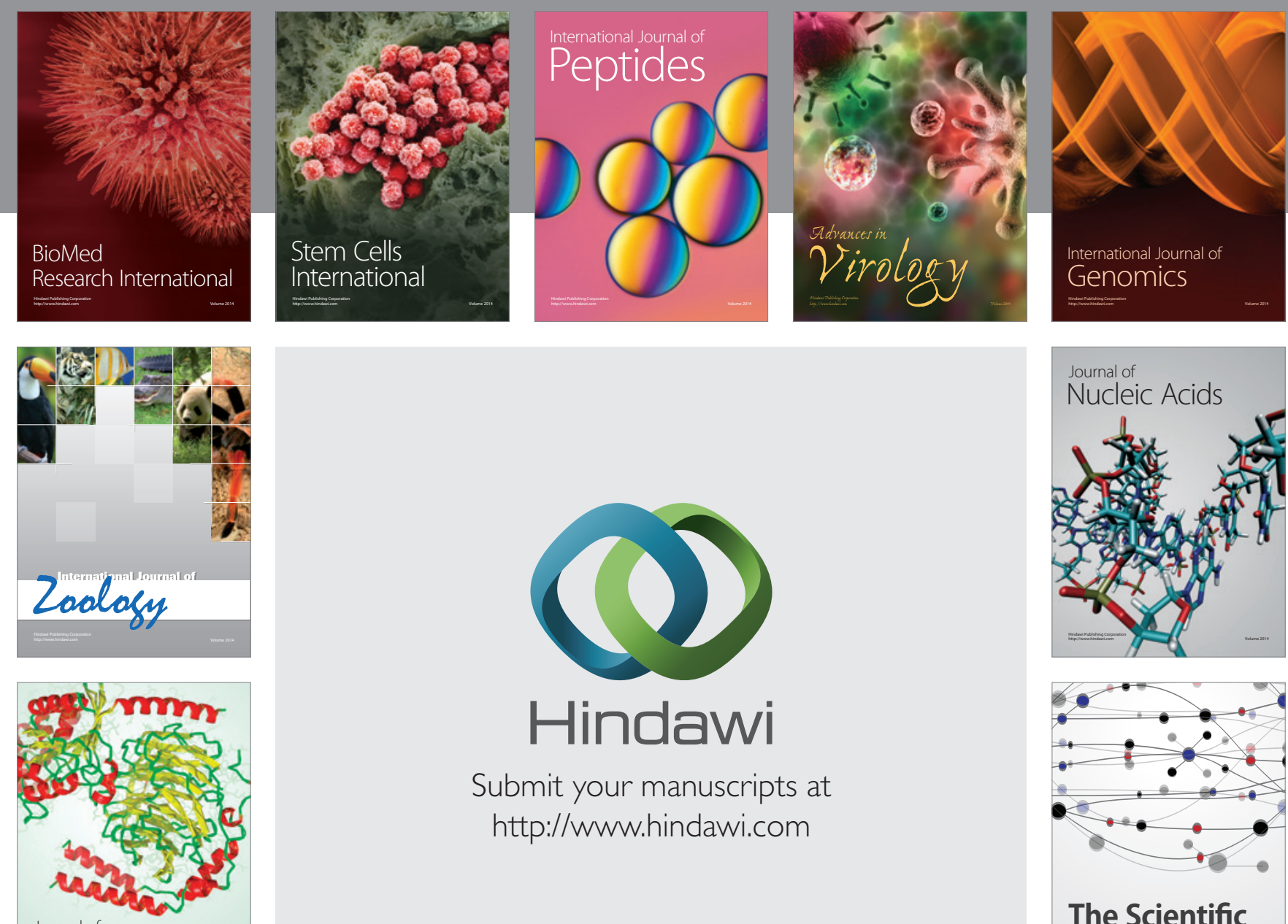

Submit your manuscripts at

http://www.hindawi.com

Journal of
Signal Transduction
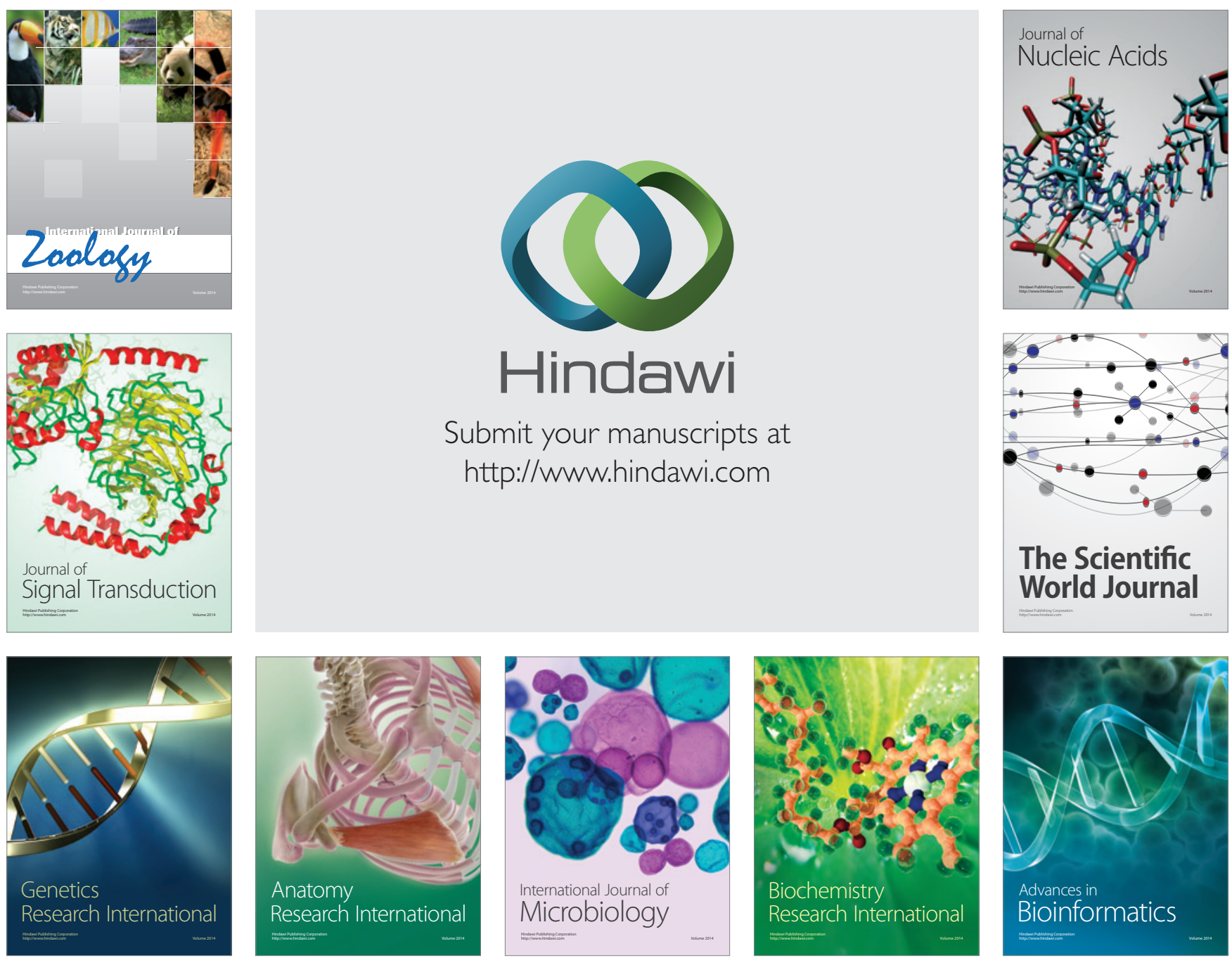

The Scientific World Journal
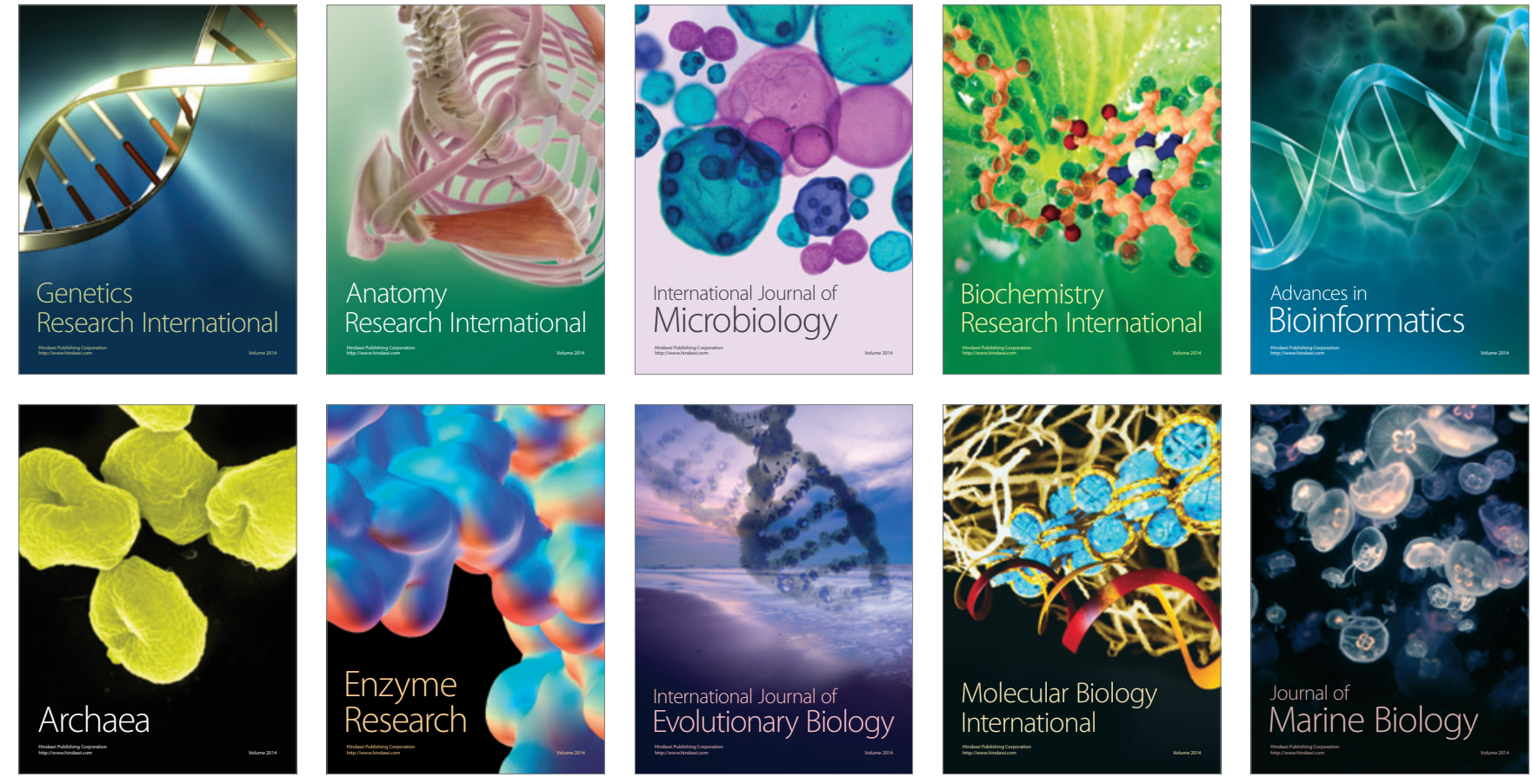